\title{
Comparative study of surface Plasmon resonance of functionalized green synthesized silver nanoparticles
}

\author{
Kamal Dhungel and Jyoti Narayan* \\ Department of Basic Science and Social Science, School of Technology, North Eastern Hill \\ University, Shillong. \\ *Email: jnarayan.nehu@gmail.com
}

Silver nanoparticles are particular interest because of distinctive properties such as good conductivity, chemical stability, catalytic and antibacterial activity [1]. In the global efforts to reduce generated hazardous waste, green chemistry and chemical processes are progressively integrating with modern developments in science and industry [2]. We use water as solvent medium, and ascorbic acid and tri-sodium citric acid as greener reducing agents. Different functionalized silver nanoparticles show different Surface Plasmon Resonance (SPR), since surface Plasmon depends on the surrounding environment, the refractive index of the surrounding medium, size and shape of the nanoparticles, the electronic interaction between the stabilizing ligands [3]. TEM pictures clearly show the morphology of the nanoparticles (Figure 1).There is a change in the Plasmon resonance as well as shape of the UV-Vis curve with different functionalization. Plasmon resonance of the synthesized nanoparticles varies with respect to $\mathrm{pH}$ of the solution (Figure 2).

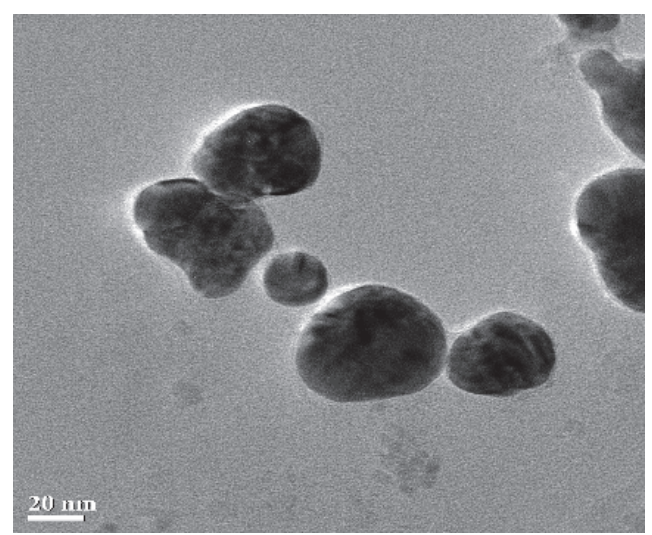

Figure 1: TEM images of silver nanoparticles synthesized by ascorbic acid
IR spectra shows the presence of functional groups $(-\mathrm{OH},-\mathrm{COOH})$ in the synthesized silver nanoparticles. The optical property of dispersed spherical particle of synthesized nanoparticle was further validated using Mie theory4. The results are in agreement with experimental techniques used.

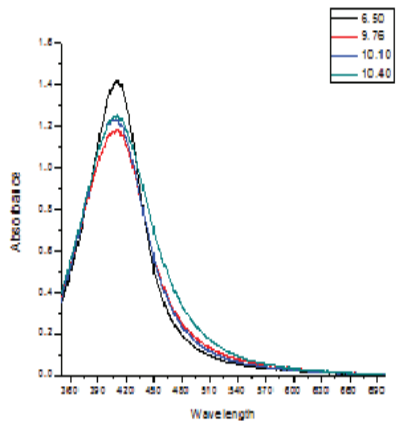

Figure 2: $\mathrm{pH}$ dependence of Surface Plasmon resonance of ascorbic acid synthesized silver nanoparticles

\section{References}

1. Zaheer Khan, Shaeel Ahemed, Al-Thabaiti, Abdullah Yousif Obaid, A.O. Al-Youbi, colloids and surfaces B: Biointerfaces 82 (2011) 513-517.

2. Virender K. Sharma, Ria A. Yngard, Yekaterina Lin, advances in colloid and interface science 145(2009), 83-96.

3. S. Link, M.A. El-Sayed, Journal of Physical Chemistry B 103 (1999), 8410.

4. Kreibig U, Vollmer M. Optical properties of metal clusters. New York: Springer. (1995) 532. 